\title{
Return of individual research results from genome-wide association studies: experience of the Electronic Medical Records and Genomics (eMERGE) Network
}

\author{
Stephanie M. Fullerton, DPhil', Wendy A. Wolf, PhD², Kyle B. Brothers, MD, \\ Ellen Wright Clayton, MD, JD³, Dana C. Crawford, PhD³, Joshua C. Denny, $\mathrm{MD}^{3}$, \\ Philip Greenland, MD4, Barbara A. Koenig, PhD ${ }^{5,6}$, Kathleen A. Leppig, MD, \\ Noralane M. Lindor, MD ${ }^{5}$, Catherine A. McCarty, PhD, MPH ${ }^{8,9}$, Amy L. McGuire, JD, PhD ${ }^{10}$, \\ Eugenia R. McPeek Hinz, MD³, Daniel B. Mirel, PhD ${ }^{11}$, Erin M. Ramos, PhD, MPH'12, \\ Marylyn D. Ritchie, PhD, MS ${ }^{13}$, Maureen E. Smith, MS, CGC4, Carol J. Waudby, MS \\ Wylie Burke, MD, $\mathrm{PhD}^{1}$ and Gail P. Jarvik, MD, PhD ${ }^{1}$
}

Purpose: Return of individual genetic results to research participants, including participants in archives and biorepositories, is receiving increased attention. However, few groups have deliberated on specific results or weighed deliberations against relevant local contextual factors.

Methods: The Electronic Medical Records and Genomics (eMERGE) Network, which includes five biorepositories conducting genomewide association studies, convened a return of results oversight committee to identify potentially returnable results. Network-wide deliberations were then brought to local constituencies for final decision making.

Results: Defining results that should be considered for return required input from clinicians with relevant expertise and much deliberation. The return of results oversight committee identified two sex chromosomal anomalies, Klinefelter syndrome and Turner

\section{INTRODUCTION}

The return of individual results from genetic research has been widely debated and discussed. There has, however, been less detailed consideration about the ways in which relevant ethical and logistical considerations change when research involves genome-wide association study (GWAS) or related whole-genome approaches, particularly as carried out in the context of large multisite research collaborations. One collaboration that has been actively grappling with the issue of individual result return is the Electronic Medical Records and Genomics (eMERGE) Network, a consortium of five institutions and two genotyping centers conducting GWAS using phenotypes derived from electronic medical records (EMRs). ${ }^{1-3}$

From its inception, the eMERGE Network incorporated active consideration of the ethical, legal, and social implications of the research, via the coordinated efforts of a consent and syndrome, as well as homozygosity for factor V Leiden, as findings that could warrant reporting. Views about returning findings of HFE gene mutations associated with hemochromatosis were mixed due to low penetrance. Review of electronic medical records suggested that most participants with detected abnormalities were unaware of these findings. Local considerations relevant to return varied and, to date, four sites have elected not to return findings (return was not possible at one site).

Conclusion: The eMERGE experience reveals the complexity of return of results decision making and provides a potential deliberative model for adoption in other collaborative contexts.

Genet Med 2012:14(4):424-431

Key Words: biorepository; context; deliberation; electronic medical records; result return

community consultation (CCC) workgroup. ${ }^{4}$ A CCC return of results subgroup recommended that the Network establish a standing return of results oversight committee (RROC) to deliberate about specific classes of findings likely to be generated by eMERGE research activities. The RROC met to consider such findings and made general recommendations to the Network, which then formed the basis for local discussion and decision making.

Here, we describe the types of findings discussed by the eMERGE RROC, the conclusions of our deliberations to date, and the ways in which specific recommendations were taken up and acted on by individual network partners. Our experience demonstrates how complicated decision making with respect to the return of individual research findings generated from whole-genome approaches is likely to be in practice, and suggests a general process that other multi-institutional genomics consortia might adopt in the future.

\footnotetext{
${ }^{1}$ University of Washington, Seattle, Washington, USA; ${ }^{2}$ Children's Hospital Boston, Boston, Massachusetts, USA; ${ }^{3}$ Vanderbilt University Medical Center, Nashville, Tennessee, USA; ${ }^{4}$ Northwestern University, Chicago, Illinois, USA; ${ }^{5}$ Mayo Clinic, Rochester, Minnesota, USA; ${ }^{6}$ University of California, San Francisco, San Francisco, California, USA; ${ }^{7}$ Group Health Cooperative, Seattle, Washington, USA; ${ }^{8}$ Marshfield Clinic Research Foundation, Marshfield, Wisconsin, USA; ${ }^{9}$ Essentia Institute of Rural Health, Duluth, Minnesota, USA; ${ }^{10}$ Baylor College of Medicine, Houston, Texas, USA; ${ }^{11}$ Broad Institute of Harvard and MIT, Cambridge, Massachusetts, USA; ${ }^{12}$ National Human Genome Research Institute, National Institutes of Health, Bethesda, Maryland, USA; ${ }^{13}$ Pennsylvania State University, University Park, Pennsylvania, USA. Correspondence: Stephanie M. Fullerton (smfllrtn@uw.edu)
} 


\section{eMERGE RROC}

The active involvement of researchers with expertise in the ethical, legal, and social implications of genomic research at all five eMERGE institutions provided an important opportunity to revisit the contours of the return of results debate in the context of biorepository-based GWAS and related genome-scale approaches. At the recommendation of the Network Steering Committee, a CCC return of results subgroup was convened in 2008 and the group met to review bioethics scholarship, policy discussion, and empirical reports of participant preferences and researcher experience with result return. A detailed literature review revealed a body of empirical data suggesting that many research participants say they would like to receive individual results from studies in which they participate; however, the degree to which there is an ethical duty to return such findings is actively debated. ${ }^{5-8}$

Based on this review, emerging national policy consensus, ${ }^{9-11}$ preliminary experience with result deliberation in related contexts, ${ }^{12,13}$ and internal debate and discussion, the CCC formally recommended the establishment of a network-wide RROC to deliberate on specific classes of findings and advise participating sites on the return of individual research results, whether generated by the primary research objectives or incidentally. To further specify the scope of the work of the RROC, the subgroup recommended limiting return only to clinically actionable findings (i.e., with potential to change immediate medical care), which the CCC agreed was the appropriate threshold for eMERGE given its use of preexisting population-based cohorts with variable consent restrictions. Given this emphasis, and under the expectation that deliberations would focus on defining the likely clinical actionability of specific classes of candidate findings, the subgroup also recommended that the RROC be composed of at least two representatives from each site: one with bioethics/policy background and one with clinical expertise. Representation from the Broad Institute and Center for Inherited Disease Research genotyping centers, as well as the National Human Genome Research Institute, was also encouraged. Finally, the CCC suggested that any recommendations made by the RROC were to be advisory, not binding, and subject to local institutional review board (IRB) oversight and approval. This was particularly important given the fact that only one of the five cohorts (Mayo Clinic) had suggested the possibility of individual result return at the time of informed consent.

The eMERGE Steering Committee formed the RROC as recommended in December 2009, and affirmed clinical actionability as the criterion for considering result return. At the first meeting of the RROC, a chair (G.P.J.) was elected and the group began meeting monthly by telephone (through December 2010) to identify relevant findings and determine their likely clinical actionability. Because none of the initial genetic associations investigated by the Network were deemed relevant to clinical care, discussion focused first on several classes of incidental findings generated by genotyping quality control, viz. sex chromosomal anomalies uncovered in the course of sex checks designed to guard against inadvertent sample mix-ups. Next, the RROC turned its attention to potentially actionable individual single-nucleotide polymorphisms (SNPs) typed on the genotyping arrays used. Identification of such SNPs was guided by a published survey of the SNPs found on major genotyping platforms that are also used as clinical tests. ${ }^{14}$ The clinical members of the RROC identified homozygosity of SNP rs6025 (R506Q, associated with factor V Leiden (FVL)) and rs1800562 (HFE C282Y, associated with hereditary hemochromatosis) as of the highest clinical relevance. The FVL-associated SNP was present only on the Illumina Infinium 1M chip used to genotype the predominantly African-American samples. This SNP was not on the $660 \mathrm{~W}$ chip used to type most eMERGE samples. Future discussion of pharmacogenetic SNPs, imputed genotypes, and copy-number variants is anticipated as part of the work of the next phase of the eMERGE collaboration.

\section{SPECIFIC FINDINGS CONSIDERED BY THE OVERSIGHT COMMITTEE}

RROC deliberations focused on genotypes associated with four diseases: Turner syndrome, Klinefelter syndrome, FVL, and hereditary hemochromatosis. Medical considerations relevant to decision making about the permissibility of return are summarized in Table 1.

\section{Sex chromosome anomalies (Turner and Klinefelter syndromes)}

In clinical care, any finding of a chromosome abnormality would typically be returned to a clinician and patient, even if the finding was incidental. For example, cytogenetic laboratories performing metaphase chromosome analysis for suspected acquired chromosome abnormalities may identify previously unrecognized constitutional sex chromosome abnormalities or acquired sex chromosome abnormalities associated with disease. ${ }^{15}$ The rationale for returning such findings is to provide patients with the opportunity for appropriate medical management. Therefore, karyotypes not requiring medical management, such as XXX (four examples of which were observed in our samples) and XYY (one observed) were not considered returnable. The standard of care for both Turner and Klinefelter syndromes involves hormone treatment and specific, syndromerelated medical screening (Table 1). For this reason, the RROC determined that chromosome anomalies associated with these disorders merited consideration for return.

When a research study includes linkage to medical recordsas with the eMERGE study-evidence of a prior diagnosis can be sought; although the EMR may be incomplete, a return of the results would not be needed if there is clear documentation of the disorder. In addition, care in interpreting GWAS data is required when sex chromosome loss is detected, particularly in older research participants, because acquired (e.g., nonconstitutional) loss occurs more commonly in older individuals. ${ }^{16}$ This is of particular importance when considering whether an older female with loss of X chromosome, particularly when mosaic, has an acquired anomaly or constitutive Turner syndrome. 
Table 1 Potentially returnable findings discussed by the eMERGE RROC

\begin{tabular}{|c|c|c|c|c|c|}
\hline Disease & Genotype & $\begin{array}{l}\text { Approximate popu- } \\
\text { lation prevalence }\end{array}$ & Pertinent features & Standard of care & RROC recommendation \\
\hline HFE hemochromatosis & $\begin{array}{l}\text { C282Y } \\
\text { rs1800562 }\end{array}$ & $\begin{array}{l}\text { Homozygote: } 1 / 500 \text { in } \\
\text { European Americans }{ }^{39}\end{array}$ & $\begin{array}{l}\text { - Homozygous genotype } \\
\text { associated with increased } \\
\text { iron absorption and } \\
\text { increased risk of } \\
\text { deposition of iron in } \\
\text { bodily tissues }{ }^{20} \\
\text { - Risk of cirrhosis, } \\
\text { hepatocellular carcinoma, } \\
\text { cardiomyopathy, } \\
\text { diabetes, and } \\
\text { hypogonadism }{ }^{40} \\
\text { - Incomplete penetrance; } \\
\text { lower in women: e.g., } \\
\text { cirrhosis estimated to } \\
\text { occur in } 10 \% \text { of male } \\
\text { homozygotes, } 1 \% \text { of } \\
\text { female homozygotes }{ }^{21}\end{array}$ & $\begin{array}{l}\text { - Testing of iron measures: } \\
\text { transferrin saturation and } \\
\text { serum ferritin }{ }^{39} \\
\text { - If iron measures elevated, } \\
\text { periodic phlebotomy } \\
\text { to prevent/reverse iron } \\
\text { overload }^{39}\end{array}$ & $\begin{array}{l}\text { Clinical benefits uncertain } \\
\text { due to low penetrance; } \\
\text { consider return to male } \\
\text { homozygotes }\end{array}$ \\
\hline
\end{tabular}

DVT, deep vein thrombosis; eMERGE, Electronic Medical Records and Genomics Network; PE, pulmonary embolism; RROC, Return of Results Oversight Committee;

VTE, venous thromboembolism.

FVL

FVL results from a mutation of the factor $\mathrm{V}$ gene, rs6025, which causes an amino acid substitution (R506Q). This results in a hypercoaguable state due to activated protein $\mathrm{C}$ resistance. Clinically this manifests as an increased risk of venous thromboembolism, including deep vein thrombosis (DVT) and pulmonary embolism (Table 1). Although the polymorphism is relatively frequent in European Americans (allele frequency $=0.05$ ), the clinical actionability of a heterozygote genotype is unclear. ${ }^{17}$ Heterozygotes have a three-to sevenfold higher than average risk of a first DVT. However, the absolute risk for heterozygotes is low (about 10\% lifetime risk of DVT) and their risk for a recurrent DVT is only slightly higher than that of DVT patients without the FVL allele. Homozygotes, in contrast, have a high lifetime risk of DVT and are likely to require treatment. ${ }^{18}$

RROC discussions attempted to balance the risk of creating "worried well" individuals among those still unlikely to develop symptoms with preventable venous thromboembolisms, and the possible benefits of disclosure. There is currently no evidence that routine FVL genotyping would change DVT risk, ${ }^{19}$ although counseling against smoking, particularly if the person is also using hormones, could theoretically reduce risk. Although some RROC members felt there was clinical value in returning a heterozygous genotype, the group ultimately agreed that such a finding would not necessitate any immediate change to medical care, and thus that it should not be included on the eMERGE list of conditions to consider returning. On the other 
hand, the group agreed that homozygosity for FVL should be considered for return to participants.

\section{HFE mutations associated with hemochromatosis}

Hemochromatosis results most often from the inheritance of two copies of the HFE mutation C282Y. This genotype is associated with increased iron absorption and a risk of deposition of iron in body tissues, including the liver, heart, skin, joints, and pancreas (Table 1). Clinical complications include cirrhosis and hepatocellular carcinoma, cardiomyopathy, diabetes, and hypogonadism. Periodic phlebotomy can prevent or reverse iron overload. Other pathologic mutations in the HFE gene have also been described and one, H63D, is associated with increased iron absorption as well; however, its penetrance is significantly lower than that of C282Y. ${ }^{20}$

A minority of people with the C282Y/C282Y genotype develop clinical disease; even among those with elevated serum iron measures, few develop complications. For example, cirrhosis is seen only among those with serum ferritin levels $>1,000 \mathrm{ng} / \mathrm{ml}$ (the normal upper limit is $300 \mathrm{ng} / \mathrm{ml}$ for men and $150 \mathrm{ng} / \mathrm{ml}$ for women), and the likelihood of people with C282Y/C282Y developing cirrhosis is estimated to be $10 \%$ or lower for men, and $<1 \%$ for women. ${ }^{21}$ However, the low penetrance must be weighed against the fact that prevention of symptomatic disease is reasonably easily achieved by periodic phlebotomy and, for the predominantly male patients who develop symptomatic disease, treatment options are limited.

In its consideration of hemochromatosis the RROC noted that expert committees have not found that population screening is warranted for this disease. ${ }^{22}$ However, a different calculus may apply when genotypes are already available. The clinical members of the committee also noted that adult family members of affected individuals are routinely screened for HFE alleles. Those who are found to be homozygous for the
$\mathrm{C} 282 \mathrm{Y}$ mutation undergo regular monitoring of their ferritin levels. Given the markedly lower expected penetrance of the homozygous genotype in females in published populationbased studies, most members of the RROC supported return of these results to males. Of 58 individuals ( 33 male) identified in eMERGE as $\mathrm{C} 282 \mathrm{Y}$ homozygotes whose medical records were evaluated, 13 (8 male) had existing diagnoses of hemochromatosis documented in the EMR. Thus, as not all diagnoses may be identified in the EMR, a minimum of $24 \%$ of males and $13 \%$ of females who were homozygotes were diagnosed at the current ages of these participants.

\section{LOCAL DELIBERATIONS SURROUNDING POTENTIALLY RETURNABLE FINDINGS}

Three of the four classes of research findings discussed by the RROC were encountered in the course of eMERGE research activities; the FVL SNP was either not assayed or genotype status was not reviewed (Table 2). At each site, EMR data were reviewed for those participants affected by the relevant genotypes. This review suggested that most participants with these findings were likely unaware of their genotype (Table 2). Local decision making regarding the necessity and/or desirability of result return differed across sites. One site (Vanderbilt) is a deidentified biobank and thus cannot return individual results. The other four sites considered the RROC recommendations in light of their original consent, as well as local advisory groups and IRB concerns. Details of local deliberations are outlined briefly below.

\section{Group Health Cooperative}

A total of 2,358 Adult Changes in Thought study ${ }^{23}$ participants (42\% male) were genotyped by Group Health/University of Washington eMERGE investigators. Of these, the results of 17 participants were relevant to disorders discussed by the RROC

Table 2 Number of participants affected by potentially returnable genotypes at each eMERGE site

eMERGE site

\begin{tabular}{|c|c|c|c|c|c|}
\hline Genetic finding & $\begin{array}{l}\text { Group Health } \\
\text { Cooperative }\end{array}$ & Marshfield Clinic & Mayo Clinic & Northwestern & Vanderbilt \\
\hline \multirow{3}{*}{$\begin{array}{l}\text { Turner syndrome, } \mathrm{XO} \text {, } \\
\mathrm{XXXO}, \mathrm{XXq-}\end{array}$} & $4 X X X O$ & 3 & 0 & 0 & 4 \\
\hline & 0 diagnosed ${ }^{a}$ & 0 diagnosed & & & $2(X 0, X X q-)$ diagnosed \\
\hline & 3 deceased & 2 infertile & & & \\
\hline \multirow{3}{*}{$\begin{array}{l}\text { Klinefelter syndrome, } \\
X X Y, X X X Y, X X Y / X Y\end{array}$} & 1 & 2 & 5 & $1(X X X Y)$ diagnosed & 2 \\
\hline & 0 diagnosed & 1 diagnosed & 1 diagnosed & & 1 diagnosed \\
\hline & 1 deceased & 1 likelyb & & & 1 likely \\
\hline $\begin{array}{l}\text { Factor V Leiden, rs6025 } \\
\text { homozygotes }\end{array}$ & Not genotyped & Not genotyped & Not genotyped & Not reviewed & Not reviewed \\
\hline $\begin{array}{l}\text { HFE mutations, } \\
\text { rs1800562 } \\
\text { homozygotes }\end{array}$ & $\begin{array}{l}12 \text { (3 male); } 1 \text { (male) } \\
\text { diagnosed }\end{array}$ & $\begin{array}{l}14 \text { (6 male); } 5 \text { (3 male) } \\
\text { diagnosed }\end{array}$ & $\begin{array}{l}14 \text { (7 male); } \\
5 \text { (3 male) diagnosed }\end{array}$ & $\begin{array}{l}6 \text { (5 male); } \\
2 \text { (1 male) diagnosed }\end{array}$ & 12 (6 male); 0 diagnosed \\
\hline
\end{tabular}

eMERGE, Electronic Medical Records and Genomics Network; EMR, electronic medical record.

aDiagnosed represents a diagnosis identified in the EMR; these data may be incomplete and represent the minimum number of preexisting diagnoses. ${ }^{\mathrm{b}} \mathrm{Although}$ there is no EMR evidence of XXY, both patients had documented abnormal testicular exams and one was treated with testosterone. 
(Table 2): four consistent with XX/XO mosaicism (none with a Turner diagnosis in the EMR), one consistent with XXY (deceased, no EMR diagnosis), and 12 HFE C282Y homozygotes. As hemochromatosis-related iron overload typically manifests earlier, HFE genotypes were unlikely to be clinically actionable for these participants, whose median age is 74 years, and hence these genotypes were not initially reviewed. However, when diagnosis rates in HFE C282Y homozygotes for other sites were higher than expected, genotypes were reviewed and 12 homozygotes (3 male; 9 deceased) were identified; one of these three males was documented to have hemochromatosis.

Local deliberation occurred at two levels: generically, as part of a consensus development discussion focused on genomic research conducted in the Group Health setting, and more specifically among the investigator team and the lead clinical geneticist at Group Health. The consensus development group ratified a careful approach in which only clinically actionable results would be returned to participants. Local investigators and the lead Group Health geneticist favored return of Turner and Klinefelter results, based on the RROC recommendation and clinical experience. The Group Health geneticist (K.A.L.) had experience with the routine return of incidental sex chromosomal anomalies to patients who have undergone bone marrow studies, in which patient responses suggest that the benefits outweigh the harms. However, for this cohort the only XXY subject was deceased. Discussion of the return of the Turner syndrome finding emphasized the fact that the four potentially affected women were of an advanced age, hence unlikely to have their care affected by the finding, and that their mosaic $\mathrm{X}$ monosomy might be acquired. The women in question had no indications in their medical records of symptoms consistent with Turner syndrome, such as short stature or infertility. Therefore, it was decided that return was not indicated due to the likelihood that these were not constitutional changes.

\section{Marshfield Clinic}

The Marshfield Clinic site genotyped and analyzed the phenotypes of 4,108 members of its Personalized Medicine Research Project (42\% male). ${ }^{24}$ Of these, 19 were identified relevant to diagnoses discussed by the RROC (Table 2): three consistent with $\mathrm{XX} / \mathrm{XO}$ (none documented in the EMR, although two had documented infertility), one consistent with XXY (no Klinefelter diagnosis, but small testes documented in the EMR) and a second with XXY/XY (Klinefelter documented in the EMR), and 14 (six male) HFE C282Y homozygotes, five previously documented with hemochromatosis (3 male).

Local deliberations focused on features of the study sample, the initial terms of informed consent, and review with the Personalized Medicine Research Project Community Advisory Group. For participants with possible Turner findings, the subjects' ages suggested that clinical care would not be improved by result return. Age was a less salient consideration for those with suspect HFE genotypes and the Marshfield Clinic IRB approved a separate study to examine the medical records of
HFE C282Y homozygotes for clinical signs and symptoms consistent with iron overload. This review revealed no symptoms suggestive of hemochromatosis among those without an EMR documented diagnosis. These findings were shared with the Community Advisory Group, together with the, then current, National Heart, Lung, and Blood Institute Guidelines for returning genetic results, ${ }^{9}$ the governing consent language suggesting that no results would be returned, and the guidelines from the Marshfield Clinic IRB. The Community Advisory Group concurred with the investigators' decision not to return HFE results. Barriers to returning genetic results that were considered in local discussions included the need to re-consent to allow result return and the logistics of how to return, including the need for genetic counseling and decision support for physicians.

\section{Mayo Clinic}

The Mayo Clinic genotyped 3,346 patients (62\% male) for eMERGE GWAS analyses. Of these, 19 were identified as relevant (Table 2): none consistent with Turner syndrome, five with findings consistent with XXY (one previously documented in the EMR), and 14 (seven male) HFE C282Y homozygotes, including five (three male) previously documented as having hemochromatosis.

The informed consent language in both the Mayo Clinic Biobank and the cardiovascular disease studies that were included in eMERGE allows for the return of individual research findings. Local deliberations at the Mayo Clinic focused on the potential return of Klinefelter syndrome findings (to date no decisions have been made about the return of HFE findings). In these local discussions, opinions were mixed, with an adult endocrinologist suggesting that the clinical benefits of hormone replacement therapy would argue in favor of return, whereas a psychiatric consultant urged great caution in considering return by investigators in light of the unknown potential consequences for each individual, and envisioned scenarios of both benefit and harm to participants, depending on individual circumstances. One eMERGE investigator was also concerned about possible discovery of non-paternity, should an affected man with children learn that his genotype generally was associated with infertility. In the wake of these discussions, the Mayo Clinic eMERGE team elected not to return this information to relevant participants. These initial internal eMERGE deliberations did provoke broader institutional discussion about return of results management, which has included the Mayo Clinic Biobank Community Advisory Board, the IRB, and other groups within Mayo.

\section{Northwestern University}

A total of 1,931 participants from the NUgene biorepository at Northwestern University ${ }^{25}$ (43\% male) were genotyped for the eMERGE study. Seven relevant individuals were reviewed. One identified as XXXY had a documented Klinefelter diagnosis. No results suggesting Turner syndrome were found. A total of six (five male) individuals were identified as HFE C282Y 
homozygotes, including two (one male) previously documented; Table 2).

Local deliberation focused on all four classes of findings discussed by the RROC, as well as the 2006 National Heart, Lung, and Blood Institute recommendations with respect to the return of individual research findings. ${ }^{9}$ Discussions were held with NUgene researchers and internal eMERGE investigators, local genetic counselors, the IRB chair who reviews NUgene applications, a hepatologist who treats patients with hemochromatosis, and the NUgene Community Advisory Committee. A number of relevant concerns were raised in the course of these deliberations, including the fact that the current NUgene consent states that no results will be returned to research participants, NUgene has a minimal ongoing relationship with participants, and the fact that return would raise a number of resource-related and logistical concerns. The varying opinions expressed during these deliberations led eMERGE investigators at Northwestern University to decide to return these (and yet to be deliberated) results in the context of a study in which participants are re-consented for result return, as well as to investigate the meaning of clinically actionable results to patients and physicians. Changes to the NUgene consent form, to allow for limited return of information, per the RROC criteria outlined earlier, are currently being discussed with relevant stakeholders, including the IRB and Community Advisory Committee.

\section{Vanderbilt University}

Vanderbilt University used for eMERGE its resource, BioVU, ${ }^{26}$ which combines irreversibly de-identified clinical information from the EMR and residual blood samples. Patients are given the opportunity to opt out of this databank; results cannot be returned, so the topic is not addressed. A total of 5,172 participants ( $40 \%$ male) were genotyped. Of these, a total of 18 individuals were identified as relevant (Table 2): four with possible Turner syndrome findings ( $1 \mathrm{X0}, 1 \mathrm{XXq}$-, and $2 \mathrm{XX} /$ $\mathrm{XO}$ ) (of which the $\mathrm{XO}$ and $\mathrm{XXq}$ - were previously documented), two consistent with XXY (one previously documented with Klinefelter and the second with a record of treatment for testosterone deficiency), and 12 (six male) HFE C282Y homozygotes (none of whom had prior documentation or EMR evidence of symptomatic hemochromatosis).

The inability to communicate individual research results in studies that use BioVU has been discussed repeatedly by the Medical Center Ethics Committee and BioVU's Community and External Ethics Advisory Boards. These groups consider this characteristic of the BioVU model to be reasonable. Vanderbilt has chosen to focus on evidence-based use of genetic testing in the clinical setting, using computer-based point-of-care decision support directed at current clinical questions.

\section{DISCUSSION}

The consortium convened a RROC to consider which eMERGE GWAS results might qualify as clinically actionable and therefore suitable to consider for return to individual participants. In this process, consideration of specific examples of results generated by network research activities helped to clarify salient issues. The committee found that whereas most findings generated from GWAS do not meet criteria for return based on clinical actionability, some may.

Unlike many forms of genomic research, the eMERGE investigators had the benefit of access to EMR information. This proved to be an important factor in decision making about specific instances of result return. In some cases, the EMR demonstrated that the clinically actionable result was already known. In other cases, health records shed light on ambiguous findings, increasing the likelihood that some findings represented acquired rather than congenital genetic changes. Decision making will likely be more complicated where there are little reliable data on whether participants are already aware of their genetic status. Although having access to EMR information was, in our experience, advantageous, we do not feel it would be appropriate to discount potential result return in the absence of such information, nor do we mean to suggest that routine access to EMR data is a prerequisite for studies that might contemplate the return of individual research findings.

Access to EMR information is only one of several aspects relevant to the return of individual research results. The informed consent process for genomic and biobank-based research often varies and may or may not have included reference to the possibility that results might be returned. For example, in the eMERGE consortium only one of the five cohorts (Mayo Clinic) had suggested the possibility of individual result return at the time of informed consent. This circumstance leads to a potential conundrum if a clinically actionable result is identified but participants were told in the informed consent process that they would not receive results. In the eMERGE CCC deliberations, the need for protection of a participant's right to refuse results was identified; however, it was also recognized that a sufficiently compelling result (i.e. one for which an investigator might reasonably conclude that risks of nondisclosure outweigh disclosure) could merit consideration as a returnable result, even if the offer of results was not anticipated at the time of consent to participate. Appropriate procedures, including robust consideration of the type of result that would meet such a standard, would need to be determined locally, in consultation with the IRB.

Another study-specific feature that has important implications for the issue of return of results is the measures taken to de-identify study data. At one eMERGE site (Vanderbilt University), this process included severing data from all identifiers-effectively anonymizing the data. Return of results is not possible in this circumstance. Because of this and other aspects of local context, including the need to involve local IRBs in the decision making about return of individual results, the eMERGE consortium determined that centralized deliberations about specific classes of results would be advisory, assisting the consortium researchers to identify results from GWAS studies that are potentially returnable, but leaving the final decision to each site. 
We believe that our experience reveals the complexity inherent to return-of-result decision making. Nonetheless, our process provides a potential model for managing such decision making in other multisite genomic studies. A centralized deliberation process, such as the one enacted here as part of the eMERGE RROC, allows for pooling of resources in a careful consideration of the results likely to be generated by studies, incorporating appropriate expertise in clinical medicine, research ethics, and genome sciences. This deliberative process can identify and justify results that could be considered for return on the basis of pre-agreed criteria such as clinical actionability. Yet, because of the many local factors that are relevant to the return of results, we believe a strong argument can be made for local control of the final decision and acknowledge that this could mean that the same finding is treated differently at different institutions. Of note, no individual results have yet been returned in the eMERGE consortium, reflecting the high bar set by our process (i.e., the focus on clinical actionability meant relatively few findings from the first phase of network activities were potentially returnable), the impact of access to medical records, and the need to balance clinical criteria with other considerations such as consent restrictions and the views of local stakeholders.

Even though the current GWAS setting is simpler than studies involving large-scale sequencing-because clinically actionable findings are infrequent - we found a range of opinion about the importance of returning specific results. Although a criterion of "clinical actionability" suggests a clear threshold for identifying results that should be considered for return, the identification of specific clinically actionable findings generated from the eMERGE studies was not straightforward. In the future, as genomic research incorporates whole-exome and wholegenome sequencing, the scope of issues to be considered will be substantially greater. With these more extensive analytical approaches, some results with urgent clinical importance will be generated, for example gene variants associated with inherited risk of colorectal cancer, and many more will be generated that are clinically important but less urgent, such as those deliberated on by our group. Most results will have uncertain clinical significance. Our experience suggests that there is a need for both research and deliberation related to return of research results, including the appropriate constitution of advisory bodies, evidence requirements for determinations of clinical validity and actionability, a consistent approach to the involvement of lay community voices, and appropriate methods for offering results to participants when findings are deemed potentially returnable.

\section{ACKNOWLEDGMENTS}

We thank the members of the eMERGE Consent and Community Consultation Return of Results work group, including Lynn Dressler (UNC Chapel Hill), Joan Henriksen Heller (Mayo Clinic), Carol Somkin (Kaiser Permanente), Holly Tabor and Ben Wilfond (Seattle Children's Research Institute), and Sue Trinidad (University of Washington). We also thank Joy Boyer and Rongling Li, of the National Human Genome Research Institute (NHGRI), for their help throughout the deliberation period.

This work was supported by the following U01 grants from the NHGRI, a component of the National Institutes of Health, Bethesda, Maryland: HG004610, AG06781 (Group Health Cooperative); HG004608 (Marshfield Clinic); HG04599 (Mayo Clinic); HG004609 (Northwestern University); HG004438 (Center for Inherited Disease Research, Johns Hopkins University); HG004424 (Broad Institute); HG004603 (Vanderbilt University, also serving as the eMERGE Administrative Coordinating Center), and R01 HG003178 (University of Minnesota). Additional support was provided by a State of Washington Life Sciences Discovery Fund award to the Northwest Institute of Genetic Medicine.

\section{DISCLOSURE}

The authors declare no conflict of interest.

\section{REFERENCES}

1. Kho AN, Pacheco JA, Peissig PL, et al. Electronic medical records for genetic research: results of the eMERGE consortium. Sci Trans/ Med 2011;3:79re1.

2. McCarty CA, Chisholm RL, Chute CG, et al. The eMERGE Network: a consortium of biorepositories linked to electronic medical records data for conducting genomic studies. BMC Med Genomics 2011;4:13.

3. McGuire AL, Basford M, Dressler LG, et al.. Ethical and practical challenges of sharing data from genome-wide association studies: the eMERGE Consortium experience. Genome Res 2011;21:1001-1007.

4. Clayton EW, Smith M, Fullerton SM, et al. Confronting real time ethical, legal, and social issues in the Electronic Medical Records and Genomics (eMERGE) Consortium. Genet Med 2010;12:616-620.

5. Bredenoord AL, Kroes HY, Cuppen E, Parker M, van Delden JJ. Disclosure of individual genetic data to research participants: the debate reconsidered. Trends Genet 2011;27:41-47.

6. Knoppers BM, Joly Y, Simard J, Durocher F. The emergence of an ethical duty to disclose genetic research results: international perspectives. Eur $J$ Hum Genet 2006;14:1170-1178.

7. Miller FA, Christensen R, Giacomini M, Robert JS. Duty to disclose what? Querying the putative obligation to return research results to participants. J Med Ethics 2008;34:210-213.

8. Ravitsky V, Wilfond BS. Disclosing individual genetic results to research participants. Am J Bioeth 2006;6:8-17.

9. Bookman EB, Langehorne AA, Eckfeldt JH, et al. Reporting genetic results in research studies: summary and recommendations of an NHLBI working group. Am J Med Genet A 2006;140:1033-1040.

10. Wolf SM, Lawrenz FP, Nelson CA, et al. Managing incidental findings in human subjects research: analysis and recommendations. J Law Med Ethics 2008;36:219-248, 211

11. Fabsitz RR, McGuire A, Sharp RR, et al. Ethical and practical guidelines for reporting genetic research results to study participants: updated guidelines from a National Heart, Lung, and Blood Institute working group. Circ Cardiovasc Genet 2010;3:574-580.

12. Kohane IS, Mandl KD, Taylor PL, Holm IA, Nigrin DJ, Kunkel LM Medicine. Reestablishing the researcher-patient compact. Science 2007;316:836-837.

13. Stack CB, Gharani N, Gordon ES, Schmidlen T, Christman MF, Keller MA Genetic risk estimation in the Coriell Personalized Medicine Collaborative. Genet Med 2011;13:131-139.

14. Johnson AD, Bhimavarapu A, Benjamin EJ, et al. CLIA-tested genetic variants on commercial SNP arrays: potential for incidental findings in genome-wide association studies. Genet Med 2010;12:355-363.

15. Wong AK, Fang B, Zhang L, Guo X, Lee S, Schreck R. Loss of the $Y$ chromosome: an age-related or clonal phenomenon in acute myelogenous leukemia/myelodysplastic syndrome? Arch Pathol Lab Med 2008;132:1329-1332.

16. Russell LM, Strike P, Browne CE, Jacobs PA. X chromosome loss and ageing Cytogenet Genome Res 2007;116:181-185. 
17. Press RD, Bauer KA, Kujovich JL, Heit JA. Clinical utility of factor V leiden (R506Q) testing for the diagnosis and management of thromboembolic disorders. Arch Pathol Lab Med 2002;126:1304-1318.

18. Rosendaal FR, Reitsma PH. Genetics of venous thrombosis. J Thromb Haemost 2009; 7(suppl 1):301-304.

19. Recommendations from the EGAPP Working Group: routine testing for Factor V Leiden (R506Q) and prothrombin (20210G>A) mutations in adults with a history of idiopathic venous thromboembolism and their adult family members. Genet Med 2011;13:67-76.

20. Allen KJ, Gurrin LC, Constantine CC, et al. Iron-overload-related disease in HFE hereditary hemochromatosis. N Engl J Med 2008;358:221-230.

21. Asberg A, Hveem K, Kannelønning K, Irgens W $\varnothing$. Penetrance of the C28Y/C282Y genotype of the HFE gene. Scand J Gastroenterol 2007;42:1073-1077.

22. Whitlock EP, Garlitz BA, Harris EL, Beil TL, Smith PR. Screening for hereditary hemochromatosis: a systematic review for the U.S. Preventive Services Task Force. Ann Intern Med 2006;145:209-223.

23. Kukull WA, Higdon R, Bowen JD, et al. Dementia and Alzheimer disease incidence: a prospective cohort study. Arch Neurol 2002;59:1737-1746.

24. McCarty CA, Nair A, Austin DM, Giampietro PF. Informed consent and subject motivation to participate in a large, population-based genomics study: the Marshfield Clinic Personalized Medicine Research Project. Community Genet 2007;10:2-9.

25. Ormond KE, Cirino AL, Helenowski IB, Chisholm RL, Wolf WA. Assessing the understanding of biobank participants. Am J Med Genet A 2009;149A:188-198.

26. Roden DM, Pulley JM, Basford MA, et al. Development of a large-scale deidentified DNA biobank to enable personalized medicine. Clin Pharmacol Ther 2008;84:362-369.

27. Hjerrild BE, Mortensen $\mathrm{KH}$, Gravholt $\mathrm{CH}$. Turner syndrome and clinical treatment. BrMed Bull 2008;86:77-93.
28. Massa G, Verlinde F, De Schepper J, et al. Trends in age at diagnosis of Turner syndrome. Arch Dis Child 2005;90:267-268.

29. Devernay M, Ecosse E, Coste J, Carel JC. Determinants of medical care for young women with Turner syndrome. J Clin Endocrinol Metab 2009;94:3408-3413.

30. Carlson M, Silberbach M. Dissection of the aorta in Turner syndrome: two cases and review of 85 cases in the literature. J Med Genet 2007;44:745-749.

31. Conway GS, Band M, Doyle J, Davies MC. How do you monitor the patient with Turner's syndrome in adulthood? Clin Endocrinol (Oxf) 2010;73:696-699.

32. Bojesen A, Gravholt CH. Klinefelter syndrome in clinical practice. Nat Clin Pract Urol 2007;4:192-204.

33. Wikström AM, Dunkel L. Klinefelter syndrome. Best Pract Res Clin Endocrinol Metab 2011;25:239-250.

34. Price WH, Clayton JF, Wilson J, Collyer S, De Mey R. Causes of death in X chromatin positive males (Klinefelter's syndrome). J Epidemiol Community Health 1985;39:330-336.

35. Sawalha AH, Harley JB, Scofield RH. Autoimmunity and Klinefelter's syndrome: when men have two X chromosomes. J Autoimmun 2009;33:31-34.

36. Brinton LA. Breast cancer risk among patients with Klinefelter syndrome. Acta Paediatr 2011;100:814-818.

37. Pomp ER, Rosendaal FR, Doggen CJ. Smoking increases the risk of venous thrombosis and acts synergistically with oral contraceptive use. Am J Hematol 2008;83:97-102.

38. Kujovich JL. Factor V Leiden thrombophilia. Genet Med 2011;13:1-16.

39. Fowler C. Hereditary hemochromatosis: pathophysiology, diagnosis, and management. Crit Care Nurs Clin North Am 2008;20:191-201, vi.

40. Alexander J, Kowdley KV. HFE-associated hereditary hemochromatosis. Genet Med 2009;11:307-313. 